\title{
Models of the anisotropy of the Earth's inner core
}

\author{
Shigeo Yoshida $\dagger$, Ikuro Sumita $\ddagger$ and Mineo Kumazawa $\S$ \\ $\dagger$ Department of Earth and Planetary Sciences, School of Science, Nagoya University, Furo-cho, \\ Chikusa-ku, Nagoya, 464-8602 Japan \\ $\ddagger$ Department of Earth and Planetary Sciences, The Johns Hopkins University, Baltimore, MD \\ 21218, USA \\ $\S$ Tono Geoscience Centre, PNC, Jorinji, Izumi, Toki, 509-5102 Japan
}

Received 2 June 1998

\begin{abstract}
Recent seismological studies have revealed that the Earth's inner core has acoustic anisotropy. Several models of the generation of the anisotropy have been proposed. Most of them attribute the anisotropy to preferred orientation of iron crystals, while the proposed mechanisms that give rise to the crystal alignment are diverse, including deformation, magnetic force and crystal growth. We review these models critically in order to suggest future directions for further development.
\end{abstract}

\section{Introduction}

The Earth's interior is composed of four layers: the crust, mantle, outer core and inner core (figure 1). The crust is the outermost skin, 5 to $10 \mathrm{~km}$ thick, and is composed of rocks of many types. The mantle extends from just below the crust down to $2900 \mathrm{~km}$ deep, and is composed of silicates. The core occupies the innermost part of the Earth, and its major component is iron. In the molten outer core, the geomagnetic field is generated through magnetohydrodynamic dynamo action. The inner core is a sphere of solid iron, and has a radius of about $1200 \mathrm{~km}$.

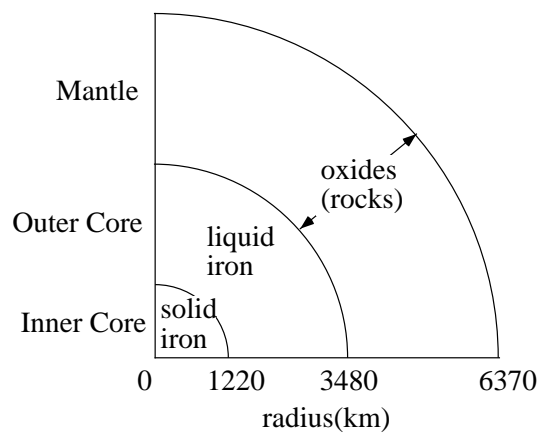

Figure 1. The interior of the Earth. The Earth is composed of four layers: the crust, mantle, outer core and inner core. The crust is not shown in the figure because it is very thin.

The core is composed mainly of iron, and has some additional impurities: about $6 \%$ of $\mathrm{Ni}$ [1] and about $10 \%$ of lighter elements such as $\mathrm{H}, \mathrm{O}, \mathrm{S}$, and $\mathrm{C}$ [2]. The light 
elements are believed to exist preferentially in the fluid outer core as compared with the solid inner core. Such composition is deduced from the comparison between the observed seismic velocity in the core, and that of pure iron measured under high-pressure and hightemperature conditions.

The pressure inside the Earth is determined well from the density distribution through the hydrostatic equation. The pressure at the boundary between the inner and outer cores, which we call the ICB (inner-core boundary) from now on, is $330 \mathrm{GPa}$. The pressure at the centre of the Earth is $360 \mathrm{GPa}$. The temperature deep inside the Earth is not known very well. The only constraint on the temperature in the core is that the ICB should be on the melting curve of iron in contact with impure iron liquid, namely, the liquidus curve.

It is believed that the inner core has been segregating from the outer core as the Earth cools throughout the Earth's history. The solidification occurs from the centre of the Earth because the gradient of the melting point of iron is steeper than the temperature gradient, which is considered to be adiabatic or less than adiabatic in the core. Therefore the solidification front of the inner core, namely, the ICB, proceeds outwards with cooling of the Earth. We stress the importance of the consideration of the inner-core history for the modelling of the inner-core anisotropy below.

\section{Seismic observation of the acoustic anisotropy of the Earth's inner core}

Acoustic anisotropy of the inner core was proposed about a decade ago on the basis of the analyses of both short-period [3] and long-period [4] seismic waves. Many earth scientists have paid attention to the anisotropy since then, because it may carry information about the dynamics in the inner core. The anisotropy has a peculiar feature; elastic waves travel faster in the polar direction than in the equatorial directions. This type of anisotropy suggests some coupling between the outer and inner cores as we discuss below. Many seismological studies have been carried out to confirm the existence of the anisotropy and to reveal its spatial distribution [5].

The important properties of the anisotropy discovered so far are:

(1) the velocities of compressional elastic waves (P waves) are larger by about 3\% in the direction of the rotation axis of the Earth than in the equatorial directions;

(2) the degree of anisotropy is small in the outermost $\sim 100 \mathrm{~km}$ of the inner core [6];

(3) the amplitude (dissipation) of the $\mathrm{P}$ waves is smaller (larger) in the direction of the rotation than in the equatorial directions $[7,8]$.

\section{The phase diagram and the acoustic anisotropy of iron}

The elastic properties of iron under high pressures and temperatures should be evaluated in order to construct a model of the seismic anisotropy.

\subsection{The phase diagram of iron}

The crystal structure of iron at inner-core conditions is important in evaluating its elastic and deformation properties. Inner-core crystals should be the phase just below the melting curve of iron at about $330 \mathrm{GPa}$, but these pressure and temperature conditions are out of reach of current experimental techniques. Therefore theoretical studies as well as experimental studies have been carried out [9] to infer the crystal structure at these conditions but the 
results are still controversial as we explain below. Figure 2 shows the phase diagram currently known.

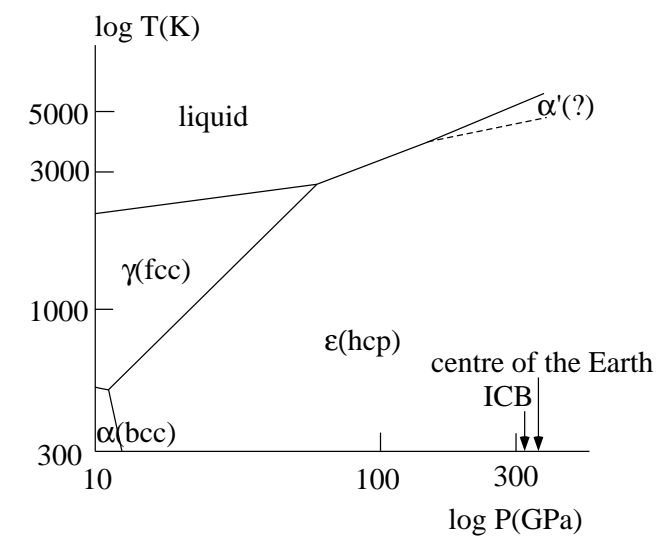

Figure 2. The phase diagram of iron. The pressures at the ICB and the Earth's centre are about $330 \mathrm{GPa}$ and $360 \mathrm{GPa}$, respectively.

Static compression experiments with diamond anvils have mainly been used to determine the phase diagram of iron. At ambient temperature, iron has bcc structure ( $\alpha$-phase) at ambient pressure, and transforms into having hcp structure ( $\epsilon$-phase) at 10-15 GPa. Mao et al [10] confirmed that the $\epsilon$-phase is stable up to about $300 \mathrm{GPa}$ by an in situ X-ray diffraction technique. Boehler [11] determined the melting curve up to about $200 \mathrm{GPa}$. He determined melting visually. He suggested a new phase just below the melting curve at high pressures, but its existence was later questioned by Yoo et al and Shen et al [12]. They determined the melting curve up to $100 \mathrm{GPa}$ with well-controlled in situ x-ray diffraction experiments, and found only $\epsilon$-phase below the melting curve above $60 \mathrm{GPa}$. It thus seems natural to assume that the inner core is composed of hcp iron from these results. However, shock experiments by Brown and McQueen [13] complicate the situation by finding an indication of solid-solid transition at $200 \mathrm{GPa}$ before melting at $240 \mathrm{GPa}$, The solid-solid transition suggests the existence of an unknown phase ( $\alpha^{\prime}$-phase) just below the melting curve at high pressures. A recurrence of the bcc phase was proposed as a possible candidate [14], but recent first-principles calculations [15, 16] showed that bcc structure is mechanically unstable with respect to a tetragonal strain at high pressures. A bct phase is suggested as an alternative candidate for the $\alpha^{\prime}$-phase [16]. This bct phase is obtained by tetragonal deformation from the bcc phase with $c / a \sim 0.9$. On the other hand, Boehler and Ross [17] interpreted the $200 \mathrm{GPa}$ transition as the onset of melting, and the $240 \mathrm{GPa}$ transition as the termination of melting. They thus argued against the existence of a new phase.

Then what is the crystal structure of the inner-core iron? We consider that the iron in the inner core has hcp structure ( $\epsilon$-phase) for the following reason. The melting point of the core iron will be much lower than that of pure iron because of impurities that are believed to exist in the core. The depression would be as much as $1000 \mathrm{~K}$ [2]. It is therefore probable that the solidus phase of core iron is a low-temperature phase ( $\epsilon$-phase) even if an unknown high-temperature phase of iron exists. Besides, if the high-temperature phase has close-packed structure, such as fcc, the discussion in this paper will not have to be altered much. 


\subsection{Elastic anisotropy of $\epsilon$-phase iron}

Elastic constants of $\epsilon$-iron under inner-core pressures were computed by first-principles calculations $[18,16]$. These theoretical calculations are valuable because laboratory measurement of elastic constants is difficult at high pressures. Despite the difficulty, Singh et al [19] and Mao et al [20] recently succeeded in determining the elastic constants at high pressures and at ambient temperature using in situ $\mathrm{x}$-ray diffraction under non-hydrostatic stresses. Singh et al reported elastic constants at $52 \mathrm{GPa}$, and Mao et al measured them at 21 pressures between 16 and $211 \mathrm{GPa}$.

Two sets of calculated elastic constants of $\epsilon$-iron are available now. Stixrude and Cohen [18] used a tight-binding Hamiltonian whose parameters are fitted to full-potential LAPW (linearized augmented-plane-wave) calculations [21] with the generalized gradient approximation for the exchange-correlation energy. Söderlind et al [16] used full-potential LMTO (linear muffin-tin orbital) calculations with the LDA (local density approximation) for the exchange-correlation energy. The two sets of elastic constants are tabulated in table 1. The two sets of results are inconsistent in terms of anisotropy. For example, the compressional velocity is faster along the $c$-axis than along the $a$-axis $\left(c_{33}>c_{11}\right)$ for Stixrude and Cohen's results, whereas the relation is opposite $\left(c_{11}>c_{33}\right)$ for the results of Söderlind et al for a volume of $7.13 \AA^{3}$. We suspect that the differences are due to the value of $c / a$ that they used; Stixrude and Cohen used an equilibrium value, while Söderlind et al used the ideal value of 1.633. The equilibrium $c / a$ value of $\epsilon$-iron is $1.57-1.60$, which has been obtained from both experiments [22,10] and theoretical calculations $[18,16]$. We believe that the equilibrium lattice parameters should be used for evaluating anisotropy since elastic anisotropy of hcp metals is known to be correlated with $c / a$ values [23]. Moreover, the experimental results of Singh et al [19] agree qualitatively with Stixrude and Cohen's in terms of anisotropy, although the experiment was carried out at a lower pressure (table 1). For example, both sets of results show that $c_{33}>c_{11}$ and $2 s_{12}-2 s_{13}-s_{11}+s_{33}<0$, where the $s_{i j}$ are the elastic compliances. The former inequality signifies that the P-wave velocity is faster along the $c$-axis than along the $a$-axis. The latter inequality is important in the model of Yoshida et al [25] of the inner-core anisotropy explained in section 4. We therefore use Stixrude and Cohen's values of elastic constants in the following.

Table 1. Elastic constants of hcp iron computed using first-principles calculations (Stixrude and Cohen [18] and Söderlind et al [16]) and measured experimentally (Singh et al [19] and Mao et al [20]). The computed values are for a volume of $7.13 \AA^{3}$, which corresponds to the inner-core density of $13 \times 10^{3} \mathrm{~kg} \mathrm{~m}^{-3}$. The experimental results of Singh et al and Mao et al are at $52 \mathrm{GPa}$ and $211 \mathrm{GPa}$, respectively. We believe that the results of Söderlind et al should not be used for the modelling of anisotropy because they used the ideal value of 1.633 for $c / a$.

\begin{tabular}{lcclc}
\hline & Stixrude and Cohen & Söderlind et al & Singh et al & Mao et al \\
\hline$c_{11}(\mathrm{GPa})$ & 1801 & 1887 & 639 & 1303 \\
$c_{12}(\mathrm{GPa})$ & 865 & 592 & 300 & 637 \\
$c_{13}(\mathrm{GPa})$ & 810 & 916 & 254 & 637 \\
$c_{33}(\mathrm{GPa})$ & 1919 & 1855 & 648 & 1302 \\
$c_{44}(\mathrm{GPa})$ & 445 & 521 & 422 & 960 \\
\hline
\end{tabular}

On the other hand, the results of Mao et al [20] at $211 \mathrm{GPa}$ (table 1) show the following three peculiar features. First, $c_{44}$ is very large. $c_{44} / c_{11}$ is more than double that for a Poisson solid, 1/3. $c_{44}$ is about triple the other rigidity, $\left(c_{11}-c_{12}\right) / 2$. Second, $c_{33}$ is almost equal to 
$c_{11}$. Third, $c_{12}$ is almost equal to $c_{13}$. The second feature means that the P-wave velocity along the $c$-axis is almost the same as that along the $a$-axis. The direction with the largest $\mathrm{P}$-wave velocity lies intermediate between the two axes due to the first feature combined with the second one. The second and the third features make $2 s_{12}-2 s_{13}-s_{11}+s_{33}$ almost zero. We do not regard these experimental results as realistic because these three features are not common to other hcp metals.

It is to be noted that the elastic constants above do not take thermal effects into account; the calculations were performed at $T=0$, and the experiments were performed at room temperature. Hence some assumptions on the thermal effect are required if we are to apply the calculated or experimental elastic constants to the inner core. Stixrude and Cohen [18] assumed that the temperature effect was incorporated by using the elastic constants at the core density, though the calculated pressure at that volume is much less than the actual pressure in the inner core. However, this assumption cannot be justified, especially for evaluating the thermal effect on the rigidity. In addition, the inner core has an anomalously high Poisson ratio of 0.44 [24]. Such a high Poisson ratio (low shear modulus) has been reproduced neither in the first-principles calculations nor in the room temperature experiment. Temperature effects would be responsible for that, because thermal vibration will smear out the potential valleys against shear deformation. Finite-temperature effects therefore should be assessed in the future, though the evaluation would require formidable computations or experimental techniques.

\section{Models of inner-core anisotropy}

Several models have been proposed to explain the acoustic anisotropy of the inner core [25-28]. Jeanloz and Wenk's idea [26] was that thermal convection in the inner core would produce crystal alignment through dislocation glide. Karato [27] argued that lattice preferred orientation would be produced during solidification of the inner core due to anisotropic paramagnetic susceptibility. Yoshida et al [25] proposed that the anisotropic heat flow in the outer core gives rise to the anisotropic growth of the inner core, which in turn induces inner-core flow to produce alignment of crystals. Bergman [28], on the other hand, pointed out that the anisotropic heat flow can directly produce alignment of crystals during solidification at the ICB. We shall examine the underlying processes below.

\subsection{The cause of seismic anisotropy}

Seismic anisotropy of the inner core can be attributed to either preferred orientation of crystals or the shape of liquid parts included in the inner core. All of the models so far assumed that the preferred orientation is the cause of the inner-core anisotropy. However, the possibility of liquid inclusions deserves attention, and we shall consider it first.

The inner core is believed to have been freezing from the outer core throughout the Earth's history. Since the outer-core iron contains some amount of impurity, freezing will occur over a range of temperature if the composition is uniform. It thus seems possible that the inner core contains a considerable amount of liquid. In addition, the impurities cause an instability of the freezing surface, and the instability produces a mushy layer on the top of the inner core [29-31]. The consideration of the mushy layer led Fearn et al [30,31] to suggest that the whole inner core may be a mixture of solid and liquid. They estimated a possible composition gradient based on thermodynamic consideration, and they concluded that the mushy layer can extend to the centre of the core. However, their assumption that impurities and heat are transported only by diffusion should not be valid, because flow would 
be generated during solidification. Loper [32] showed that convective transfer reduces the mushy layer thickness to about $300 \mathrm{~m}$. Sumita et al [33] demonstrated the importance of solid flow, which results in compaction, in the long history of the growth of the inner core. They showed that most of the liquid would have been squeezed out by deformation of the solid matrix. It is consequently unlikely that the inner core contains a considerable amount of liquid. Nevertheless it is still possible that some liquid inclusions with high concentration of impurities remain in the inner core if paths for liquid to escape are closed [34].

The anisotropy in attenuation mentioned in section 2 is an important constraint for determining whether fluid inclusions are the cause of seismic anisotropy, because fluid inclusions reduce the seismic velocity and increase the attenuation [35]. Souriau and Romanowicz [8] argued against fluid inclusions because their analyses show that $\mathrm{P}$ waves that travel in the faster direction are strongly attenuated. Hence lattice preferred orientation should be a dominant mechanism of the observed seismic anisotropy.

The anisotropy caused by crystal alignment of $\epsilon$-iron is simple. If we take the elastic constants of Stixrude and Cohen, the $c$-axes should align in the direction of the rotation axis. In the following discussion, we shall deal only with this lattice preferred orientation mechanism and see how the alignment can be achieved.

\subsection{How can the inner-core crystals recognize the direction of the Earth's rotation axis?}

The inner-core anisotropy has the peculiar feature that the $\mathrm{P}$ waves travel fast in the direction of the rotation of the Earth. In other words, the inner-core crystals recognize somehow the direction of the rotation axis. There are two ways that this can be accomplished. One is through direct effects, and the other is through influences of the outer core.

Some possible direct effects are proposed by Stevenson [36]. He proposed that tidal stress, rotational relaxation or the gravitational force imposed by the heterogeneity of the mantle could be a possible source of stress that can give rise to the preferred orientation.

In most of the models proposed so far [27, 25, 28], inner-core crystals align under influences of the outer core. Outer-core fluid flow is strongly influenced by the Coriolis force as is evident from the geomagnetic poles pointing almost to the direction of the rotation.

Yoshida et al [25] and Bergman [28] considered that the anisotropy in heat flow would affect the alignment of crystals. The convection in the outer core will take the form of rolls aligned in the direction of rotation due to the Coriolis force. This tendency persists even when the magnetic field is substantial [37]. This type of convection transports heat in the cylindrically radial direction [38]. Yoshida et al [25] pointed out that the anisotropic heat transfer will result in the anisotropic growth of the inner core, which is freezing from the outer core. The anisotropic growth produces an oblate inner core. The ellipticity does not become large because a flow will be produced to make the inner core spherical. The stress accompanying the flow thus produced aligns crystals in the inner core through recrystallization (figure 3 , section 4.4). They found that $c$-axes of hcp crystals align in the inner core, except near the ICB, consistently with observations. The theory has the problem that the flow may be too weak to produce the anisotropy in a sufficiently short time, as they pointed out in their paper.

Bergman [28] proposed that the anisotropic heat flow would directly induce crystal alignment during solidification. His model relies on the direction of the heat flow being cylindrically radial. However, we believe that the direction of the heat flow in the boundary layer would be spherically radial on average and laterally inhomogeneous, although it would be cylindrically radial in the body of the outer core. It is possible that the inner core grows 


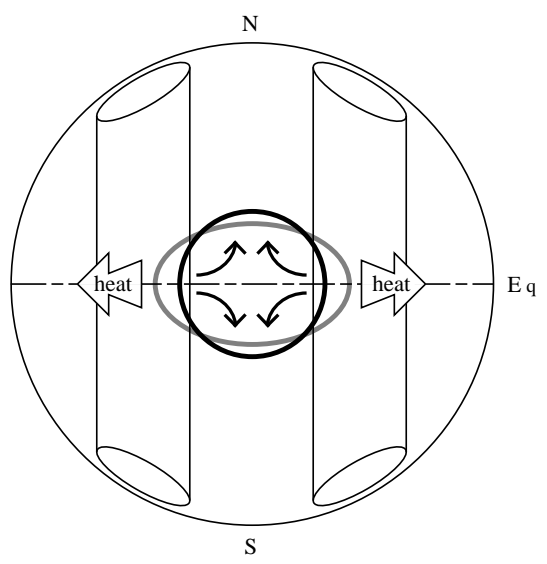

Figure 3. A conceptual picture of the Yoshida et al model [25]. Columnar convection formed under the strong influence of the Coriolis force transports heat more in the equatorial region than in the polar regions. The anisotropic heat transfer gives rise to anisotropic growth of the inner core, which, in turn, produces flow in the inner core to keep the state of isostatic equilibrium.

only in the equatorial region. In that case, spherically radial and cylindrically radial heat flow directions do not differ much. However, the equatorial growth should be accompanied by the flow as envisaged in the Yoshida et al model, and the flow would modify the alignment generated on the inner-core surface.

Karato [27] proposed that the magnetic field would align inner-core crystals during solidification. In dynamo theories, a magnetic field can be divided into poloidal and toroidal parts. Magnetic field generation is often interpreted as the result of the interaction of poloidal and toroidal fields. An axisymmetric part of the toroidal field encircles the inner core in the east-west direction, and it is considered to be stronger than the poloidal field, or at least of the same magnitude. Karato's theory predicts that the $c$-axis aligns in the magnetic field direction, which is probably in the east-west direction. Thus his theory leads to an anisotropy inconsistent with observations. This is partly due to his assumption that the compressional wave velocity is lower along the $c$-axis than along the $a$-axis, which would not hold as we explained above. A strong poloidal field is a possibility for accommodating his theory to observations, but it is unlikely according to dynamo theories.

McSweeney et al [39] mentioned the possibility of solidification in the mushy layer (section 4.1) under the influence of a magnetic field, but they did not suggest any physical mechanism for the magnetic interaction. They also mentioned the crystal growth under the influence of the flow field. The east-west flow would be prevalent in the outer core, and may influence the crystal orientation.

In their model of thermal convection as the cause of seismic anisotropy, Jeanloz and Wenk [26] did not propose any mechanism by which the convection pattern in the inner core aligns in the direction of the rotation axis. This point is discussed further, below.

\subsection{The possibility of the convection in the inner core}

Thermal convection would be important in generating preferred orientation if it exists. Jeanloz and Wenk [26] proposed that thermal convection is powered by radiogenic heating. However, this is unlikely because radioactive nuclei would be partitioned more into the liquid outer core than into the solid inner core. Moreover, analogy with iron meteorites 
implies that the absolute amount of radiogenic sources would be small. Yoshida et al [25] examined the possibility of the inner-core convection being driven by primordial heat, and concluded that this is also unlikely due to a large thermal conductivity of the inner-core iron. Nevertheless, thermal convection in the inner core cannot be totally rejected, because of the uncertainty of the physical parameters.

If there is convection in the inner core, the axial nature of the inner-core anisotropy suggests coupling between the inner- and outer-core convections for the following reason. Thermal convection strongly affects crystal alignment. Hence the axial alignment of the inner-core crystals implies that the convection in the inner core should be affected by the Earth's rotation. However, the thermal convection in the inner core cannot be affected by the Coriolis force because the viscous force is much stronger. Therefore the inner-core convection should be thermally coupled with the outer-core convection to produce the axial anisotropy as Yoshida et al [25] suggested. The pattern of convection would be of $Y_{2}^{0}$ type, i.e., 'downwelling' at equatorial regions and upwelling at polar regions due to the anisotropic heat transfer in the outer core. In view of the thermal interaction discussed above, the degree-one convection cell given a priori in some models [26, 41] would not exist. Even if the influence of the outer core is absent, the degree-one cell would not prevail, because convective patterns are not simple except near the critical Rayleigh number [40].

\subsection{The mechanism of crystal alignment}

The mechanisms proposed so far for aligning crystals in the inner core are diverse. They can be classified in two groups in terms of the location at which the alignment is generated. One group has the alignment at the solidification front, i.e., on the inner-core surface. The other has the alignment generated inside the inner core. Mechanisms of the latter type seem likely, since seismic anisotropy is weak in the shallow part of the inner core [6].

First we review the mechanisms of the alignment occurring during deposition of iron crystals on the inner-core surface. There are several mechanisms:

(1) gravitational settling of needle-shaped or planar crystals [39];

(2) crystal growth in a flow field [39];

(3) growth of crystals that have anisotropic magnetic susceptibility in a magnetic field [27]; and

(4) crystal growth in a directional heat flow [28].

Of these, (1) and (2) have only been mentioned without quantitative estimates, while (3) and (4) have been proposed with some quantitative estimates.

Karato [27] presented the third mechanism of magnetic anisotropy. This mechanism requires a condition which may not hold in the inner core as he pointed out himself. The melting temperature should be considerably depressed by impurities in order for the magnetic susceptibility to be anisotropic, and the required depression may be too large. Moreover, he considered only Brownian motion for disturbances which can destroy magnetic alignment. This led him to conclude that preferred orientation would develop for crystals larger than $\sim 10 \mu \mathrm{m}$. However, other physical effects are much larger for crystals large enough for thermal disturbances to be negligible, as Stevenson [36] pointed out. In addition, theoretical work on the magnetism of iron crystals $[42,43]$ shows that the effect of a magnetic field on the crystal alignment would be negligible under core conditions.

Bergman [28] performed an experiment using a lead-tin alloy (solder) to show that a specific crystallographic axis aligns in the direction of the heat flow. He proposed that the $\langle 210\rangle$ axis aligns in the cylindrically radial direction in the inner core. However, Brito 
[44], on the basis of solidification experiments on liquid gallium, reports that directional solidification is dictated primarily by the orientation of the initial germ crystal and does not correlate with the cooling direction or the ambient magnetic field. The discrepancies above could be due to the difference in the analogue materials used; dendritic crystal growth is enhanced for alloy more than for pure metal due to compositional supercooling. Laboratory experiments are important, in that microscopic processes relevant in crystal growth are realized, but caution should be exercised when extrapolating the results to the inner core, with considerably different spatial and temporal scales.

Bergman's model has the interesting feature that the difference between the travel times in the polar and equatorial directions is small at the top of the inner core although the crystal alignment is generated at the inner-core surface. This is because an axis with a slow P-wave velocity aligns in the cylindrically radial direction. Seismic rays cross the slow direction in the shallow part irrespective of ray directions except near the poles. Though we doubt his assumption about the heat flow direction (section 4.2), this type of crystal alignment remains as a candidate for the origin of the inner-core anisotropy.

Next we turn to alignment mechanisms that occur inside the inner core. There are two mechanisms that can change crystal orientations: (1) inclining of crystal axes by dislocation glide [26]; and (2) growth of crystals that has a favourable orientation in terms of some kinds of energy, such as elastic strain energy [25], plastic strain energy (dislocation energy), or magnetic energy.

These alignment mechanisms are related to the deformation mechanism. In the region of dislocation creep, the first mechanism of dislocation glide tends to prevail, whereas in the region of diffusion creep, the second mechanism of recrystallization prevails. The dominant deformation mechanism is determined by the magnitude of the stress and the grain size. When the stress is large, dislocation creep dominates because the large dislocation density facilitates deformation. When the stress is small, diffusion creep dominates because the dislocation density is small and diffusion of point defects governs the deformation. Diffusion creep is dominant when the grain size is small. Yoshida et al [25] argued against thermal convection in the inner core (section 4.3), and considered that the flow is induced by the anisotropic growth of the inner core (section 4.2). The stress in their model is consequently small because the growth rate of the inner core is considered to be of the order of $0.1 \mathrm{~mm}$ per year, which is derived from thermal history calculations. They estimated the stress to be $10^{4} \mathrm{~Pa}$, and the grain size to be about $5 \mathrm{~m}$. Under these conditions, recrystallization would be the mechanism generating preferred orientation. Jeanloz and Wenk [26], on the other hand, assumed dislocation creep with dislocation glide as the mechanism generating preferred orientation. However, the values of stress and viscosity that they used are not internally consistent. A third deformation mechanism, Harper-Dorn creep, may dominate the deformation in the inner core. Harper-Dorn creep is a kind of dislocation creep under low stresses with stress-independent dislocation densities. It may invalidate the model of Yoshida et al, but the nature of the creep is not well understood yet.

Recrystallization is the reorganization process of the grain boundaries. In the model of Yoshida et al [25], the driving force for grain boundary migration is the difference between the strain energies of the individual crystals that have different orientations with respect to the ambient stress field. The orientation which minimizes the strain energy is the one which results in the preferred orientation. Yoshida et al calculated the preferred orientation for both hcp and fcc crystals, and found that the two crystal structures explain the observed anisotropy of the inner core equally well.

However, the main driving forces of grain boundary migration are, in general, interface energy and dislocation energy difference between worked and virgin grains [48]. Yoshida 
et al argued that preferred orientation due to strain energy will emerge after a long time through the dynamic recrystallization process. This point should be investigated further.

In relation to the deformation mechanism, estimation of the viscosity is very important. Yoshida et al estimated the viscosity to be of the order of $10^{21} \mathrm{~Pa} \mathrm{~s}$ for the NabarroHerring creep mechanism. They also gave the constraint that the viscosity is at least of the order of $10^{16} \mathrm{~Pa}$ s to explain the seismic $Q$. On the other hand, Buffett [45] showed that the viscosity should be either above $10^{20} \mathrm{~Pa} \mathrm{~s}$ or below $10^{16} \mathrm{~Pa} \mathrm{~s}$ in order that the inner core rotates at an angular velocity different from that of the mantle [46]. We cannot derive a definite conclusion from these figures, but more reliable estimates of the viscosity would give constraints on the deformation mechanism and possibly the mechanism of lattice preferred orientation.

Estimation of the grain size is important in determining the viscosity and the mechanism of preferred orientation. Yoshida et al [25] estimated the grain size to be about $5 \mathrm{~m}$ from the empirical relation for the dynamic equilibrium between grain growth and grain refinement during deformation. On the other hand, Bergman [47] estimated it to be of the order of $100 \mathrm{~m}$, assuming that the seismic $Q$ is totally due to scattering at crystal interfaces. Though his estimate relies on his model of anisotropy [28], it gives a rough idea about the effect of scattering at crystal interfaces. If his estimate is correct, it implies that the viscosity is large and that dislocation glide can become the mechanism for preferred orientation.

\section{Concluding remarks}

In order to check the validity of the models proposed, it is necessary to compare with and predict the seismic structure of the inner core. It should first be noted that acoustic wave propagation in an anisotropic medium involves a complication, because the propagation of the wave front (i.e., the group velocity) does not coincide with the direction of the wave vector (i.e., the phase velocity), and the propagation direction shifts towards the fastpropagating direction relative to the wave-vector direction [49]. Incorporation of this effect can be important in the evaluation of travel time and amplitudes. An example of a simplified version of such calculation [50] shows that this effect is insufficient to explain the observed amplitude anomaly mentioned in section 2 . It can also be shown that the shear waves, which have not been observed yet, show a stronger anisotropy as compared with the compressional wave, and can result in differences in travel time as large as 20 seconds and focusing in particular regions. Developments of the method for analysing seismic observations are necessary if we are to search for unidentified shear waves and their polarization anisotropy.

In this paper we have focused primarily on the elastic anisotropy of the inner core, and reviewed the models presented hitherto. We believe that the presence of anisotropy is related not only to the problem of the elastic constants of possible hcp iron in the inner core, but also to the large-scale dynamics involving the entire core. We emphasize the importance of considering the growth history of the inner core, since the inner core is a cumulative result of the cooling Earth, and because preferred orientation would have a memory of the past. For example, our attempt to calculate the stratigraphic structure of the inner core [25], revealing essentially cylindrical strata, can have relevance to the reported observation that the degree of anisotropy is large for the region near the rotational axis [51].

There are other important observed properties of the inner core, such as the presence of lateral heterogeneity of low degree near the surface of the inner core [52], the possible presence of a tilt in the anisotropy axis relative to the rotational axis [53], and the presence of inner-core super-rotation relative to the outer core [46]. Needless to say, a successful model is one which can show how these properties are related to each other. 


\section{Acknowledgments}

We are grateful to David Stevenson and an anonymous reviewer for their helpful comments. We thank Russell Hemley for sending us a preprint.

\section{References}

[1] See, e.g.,

Ringwood A E 1966 Geochim. Cosmochim. Acta 30 41-4

Ringwood A E 1977 Geochem. J. 11 111-35

[2] Jeanloz R 1990 Annu. Rev. Earth Planet. Sci. 18 357-86 and references therein

[3] Morelli A, Dziewonski A M and Woodhouse J H 1986 Geophys. Res. Lett. 13 1545-8

[4] Woodhouse J H, Giardini D and Li X-D 1986 Geophys. Res. Lett. 13 1549-52

[5] Song X 1997 Rev. Geophys. 35 297-313 and references therein

[6] Shearer P M 1994 J. Geophys. Res. 99 19647-59

Song X D and Helmberger D V 1995 J. Geophys. Res. 100 9805-16

[7] Creager K 1992 Nature 356 309-14

[8] Souriau A and Romanowicz B 1996 Geophys. Res. Lett. 23 1-4

Souriau A and Romanowicz B 1997 Phys. Earth Planet. Inter. 101 33-47

[9] Anderson O L and Duba A 1997 J. Geophys. Res. $10222659-69$ and references therein

[10] Mao H-K, Wu Y, Chen L C, Shu J F and Jephcoat A P 1990 J. Geophys. Res. 95 21737-42

[11] Boehler R 1993 Nature 363 534-6

[12] Yoo C S, Akella J, Campbell A J, Mao H-K and Hemley R J 1995 Science 270 1473-5 Shen G, Mao H-K, Hemley R J, Duffy T S and Rivers M L 1998 Geophys. Res. Lett. 25 373-6

[13] Brown J M and McQueen R G 1982 High-Pressure Research in Geophysics ed S Akimoto and M H Manghnani (Tokyo: Centre for Academic Publications) pp 611-23

Brown J M and McQueen R G 1986 J. Geophys. Res. 91 7485-94

[14] Ross M, Young D A and Grover R 1990 J. Geophys. Res. $9521713-6$ Matsui M and Anderson O L 1997 Phys. Earth Planet. Inter. 103 55-62

[15] Stixrude L and Cohen R E 1995 Geophys. Res. Lett. 22 125-8

[16] Söderlind P, Moriarty J A and Wills J M 1996 Phys. Rev. B $5314063-72$

[17] Boehler R and Ross M 1997 Earth Planet. Sci. Lett. 153 223-7

[18] Stixrude L and Cohen 1995 Science 267 1972-5 Cohen R E, Stixrude L and Wasserman E 1997 Phys. Rev. B 56 8575-89

[19] Singh A K, Mao H-K, Shu J and Hemley R J 1998 Phys. Rev. Lett. 80 2157-60

[20] Mao H-K, Shu J, Shen G, Hemley R J, Li B and Singh A K 1998 Nature submitted

[21] Stixrude L and Cohen R E 1994 Phys. Rev. B 50 6442-5

[22] Jephcoat A P, Mao H-K and Bell P M 1986 J. Geophys. Res. 91 4677-86

[23] Wenk H R, Takeshita T, Jeanloz R and Johnson G C 1988 Geophys. Res. Lett. 15 76-9

[24] See, e.g., Dziewonski A and Anderson D 1981 Phys. Earth Planet. Inter. 25 297-356

[25] Yoshida S, Sumita I and Kumazawa M 1996 J. Geophys. Res. 10128 085-103

[26] Jeanloz R and Wenk H-R 1988 Geophys. Res. Lett. 15 72-5

[27] Karato S 1993 Science 262 1708-11

[28] Bergman M I 1997 Nature 389 60-3

[29] Loper D E and Roberts P H 1981 Phys. Earth Planet. Inter. 24 302-7

[30] Fearn D R, Loper D E and Roberts P H 1981 Nature 292 232-3

[31] Loper D E and Roberts P H 1982 Stellar and Planetary Magnetism ed A M Soward (New York: Gordon and Breach) pp 297-327

[32] Loper D E 1983 Geophys. Astrophys. Fluid Dyn. 25 139-55

[33] Sumita I, Yoshida S, Kumazawa M and Hamano Y 1996 Geophys. J. Int. 124 502-24

[34] Sumita I, Yoshida S, Kumazawa M and Hamano Y 1995 The Earth's Central Part: Its Structure and Dynamics ed T Yukutake (Tokyo: Terra Scientific) pp 231-61

[35] Crampin S 1984 Geophys. J. Int. 76 135-45

Peacock S and Hudson J A 1990 Geophys. J. Int. 102 471-84

[36] Stevenson D J 1996 EOS Trans. Am. Geophys. Union (1996 Western Pacific Geophysics Mtg Suppl.) 77 W139

[37] Glatzmaier G A and Roberts P H 1995 Nature 377 203-9 
Glatzmaier G A and Roberts P H 1995 Phys. Earth Planet. Inter. 91 63-76

Kageyama A, Sato T and the Complexity Simulation Group 1995 Phys. Fluids 2 1421-31

Kageyama A and Sato T 1997 Phys. Fluids 4 1569-75

[38] Zhang K 1991 Phys. Earth Planet. Inter. 68 156-69

[39] McSweeney T J, Creager K C and Merrill R T 1997 Phys. Earth Planet. Inter. 101 131-56

[40] Weber P and Matchetel P 1992 Geophys. Res. Lett. 19 2107-10

[41] Baumgardner J R, Tomé C N, Lebensohn R and Wenk H R 1997 EOS Trans. Am. Geophys. Union (1997 Fall Mtg Suppl.) 78 F458

[42] Sherman D 1996 EOS Trans. Am. Geophys. Union (1996 Western Pacific Geophysics Mtg Suppl.) 77 W132

[43] Steinle-Neumann G, Stixrude L and Cohen R 1997 EOS Trans. Am. Geophys. Union (1997 Fall Mtg Suppl.) 78 F486

[44] Brito D 1998 Thesis Ecole Normale Supérieure, Paris, and Johns Hopkins University, Baltimore, MD

[45] Buffett B A 1997 Nature 388 571-3

[46] Song X and Richards P 1996 Nature 382 221-4

[47] Bergman M I 1998 Geophys. Res. Lett. 25 1593-6

[48] Karato S 1988 Phys. Earth Planet. Inter. 51 107-22

[49] Musgrave M 1970 Crystal Acoustics (San Francisco, CA: Holden-Day)

[50] Sumita I 1997 Thesis University of Tokyo

[51] Romanowicz B, Li X-D and Durek J 1996 Science 274 963-6

[52] Kaneshima S 1996 Geophys. Res. Lett. 23 3075-8

Tanaka S and Hamaguchi H 1997 J. Geophys. Res. 102 2925-38

[53] Su W and Dziewonski A 1995 J. Geophys. Res. 100 9831-52 